\section{Aufzeigen von Optimierungspotentialen}

Wie bereits erwähnt wurde, ist ein wesentlicher Bestandteil der Produktbilanz nach IÖW/ecco die Handlungsorientierung. Deshalb werden über die Bewertung der Umweltwirkungen nach den oben genannten quantitativen und qualitativen Kriterien hinaus Optimierungspotentiale aufgezeigt. Das können Empfehlungen zur Gestaltung der Rahmenbedingungen sein. Will man bspw. auf Messen Mehrweggeschirr einsetzen, ist darauf zu achten, daß das Geschirr gründlich vorgesäubert wird, bevor es in die Spülmaschine kommt, um die Wasserbelastun- gen zu vermindern. Weiterhin sollten zur Verringerung des Energieverbrauchs bei der Wassererwärmung die Messestände mit Warmwasseranschlüssen für die Spülmaschinen versehen werden, um nur einige Beispiele zu nennen. Über Empfehlungen hinaus können Szenarien erstellt und im Hinblick auf ihre Umweltwirkungen durchgerechnet werden. Damit werden komplexere Sachverhalte und Zusammenhänge aufgedeckt und nachvollziehbạr gestaltet.

Bisher noch ungelöst ist die Frage, wie über Produktbilanzergebnisse kommuniziert werden soll und kann. Auf der einen Seite ist die Veröffentlichung durch Geheimhaltungsklauseln ein- geschränkt, auf der anderen Seite ist es bisher nicht gelungen; eine einheitliche Form zu finden, die Ergebnisse für verschiedene Interessengruppen kurz, verständlich und nachvoliziehbar aufzubereiten. Diese Fragestellungen sollen in verschiedenen Forschungsprojekten des IÖW aufgegriffen und gelöst werden.

Kathrin Ankele, Berlin

(1) Biet, J. et al. (1992): Ökobilanzen für Produkte. Bedeutung - Sachstand - Perspektiven. Umweltbundesamt $38 / 92$, Berlin

\title{
Die Anwendung von Produktbilanzen
}

Die Geschichte der Entwicklung und Anwendung von Produktbilanzen reicht bis in die 70er Jahre zurück. Allerdings wurden damals noch nicht die heute im deutschen Sprachraum am häufigsten verwendeten Begriffe »ProduktÖkobilanz « und »Produktlinienanalyse « - die beide erst Ende der 80er Jahre geprägt wurden und der international gebräuchlichste Begriff "Life-Cycle-Assessment « (LCA), der auch erst in den letzten Jahren den Durchbruch geschafft hat, benutzt. Die in der »Frühzeit« der Produktbilanzierung verwendeten Begriffe sind von allen typischen Merkmalen eines aufstrebenden Arbeitsgebietes gekennzeichnet:

- Verschiedene Autoren »kreieren « eigene Namen für ein Konzept, unabhängig davon, ob dieses Konzept schon einen Namen hat.

- Ein Konzept gleichen Namens wird von den verschiedenen Autoren unterschiedlich gehandhabt und ausgefüllt.

Mit Fug und Recht kann man die Situation der Frühzeit der Produktbilanzierung als eine Zeit einer gewissen $\gg$ babylonischen Sprachverwirrung « und eines großen Methodenpluralismus kennzeichnen. Diese Zeit scheint heute überwunden zu sein.

Eine empirische Auswertung der ProduktbilanzStudien der letzten Jahre kann sich jedoch nicht ausschließlich auf den seit kurzem entwickelten Standard beschränken, denn damit würden eine große Anzahl von Ärbeiten unberücksichtigt bleiben. Vielmehr muß auch die »Frühzeit« der Produktbilanzierung beachtet werden. Dies bedeutet, daß mitunter Arbeiten erfaßt werden, die aus heutiger Sicht wahrscheinlich anders beurteilt werden, zum Zeitpunkt ihrer Erstellung jedoch große Beachtung gefunden haben. Die empirische Basis beschränkt sich auf die uns aus der einschlägigen Diskussion bekannt gewordenen Studien in der eben erläuterten weiten begrifflichen Abgrenzung. Eine Anzahl von Arbeiten dürften dabei nicht berücksichtigt worden sein:

- Zum einen Arbeiten, die von Unternehmensberatungsbüros für Privatunternehmen er- stellt werden und von keiner der daran beteiligten Seiten publiziert werden. Beispielsweise hat die amerikanische Firma Franklin Associates bis Ende 1991 rund 70 Studien erstellt, von denen aber nur vier bekannt geworden sind

- Zum anderen Arbeiten, die im Ausland erstellt worden sind und aufgrund der Sprache nicht in die internationale (englischsprachige) Diskussion eingegangen sind.

Gleichwohl ist die empirische Basis von 200 Arbeiten ausreichend, um diese nach einigen Merkmalen auszuwerten. Die Arbeiten, auf die wir uns beziehen, sind im einzelnen in einer $\mathrm{Bi}$ bliographie aufgelistet, die wir erstellt haben und zweimal jährlich aktualisieren. Diese Bibliographie ist als IÖW-Schriftenreihe 70/94 in einer Neuauflage erschienen.

Produktbilanz-Studien werden seit 1974 erstellt; sie nahmen eine langsame Entwicklung bis Ende der 80er Jahre. Erst Ende der 80er Jahre hat die »Hochzeit « eingesetzt. 1992 und 1993 hat sich ein gewisser Abschwung gezeigt, der möglicherweise auf die konjunkturelle Entwicklung zurückzuführen ist. Als eine Trendwende sollte dieser Rückgang an Studien jedoch nicht interpretiert werden. Produktbilanzen werden hauptsächlich bisher in der Bundesrepublik und in der Schweiz erstellt. Danach folgen die USA. Relativ wenig Bedeutung haben sie bisher in den südeuropäischen Mitgliedsstaaten der Europäischen Union, wenngleich in İtalien wohl auch bedingt durch die Absicht, den $\gg$ Anschluß« nach Mitteleuropa zu schaffen - das Thema an Bedeutung gewinnt.

Die Themen bzw. Untersuchungsobjekte von Produktbilanzen konzentrieren sich auf einige Bereiche: Dominant dabei ist, wie die Tabelle zeigt, der Bereich der Verpackungen, auf den immerhin rund $40 \%$ aller Studien entfallen. Der Grund für die Dominanz dieses Bereiches liegt in der Kritik an den Verpackungen selbst, wie er speziell von Umweltorganisationen geübt worden ist. Unternehmen reagierten darauf mit der Erstellung von Studien, die sowohl der Recht- fertigung wie auch der echten Verbesserung der Verpackungen dienten.

Neben den Verpackungen sind die Bereiche der Hygiene- und Reinigungsprodukte (darunter speziell auch Windeln), der Kunststoffgranulate sowie der Baumaterialien bedeutsam. Dieser Darstellung ist jedoch nicht die Entwicklung der Themen zu entnehmen. Eine Analyse der Entwicklung der letzten Jahre zeigt, daß die Bedeutung des Verpackungsbereiches abgenommen hat: Wurden 1988/89 noch 53\% aller Arbeiten zu diesem Thema gemacht, so reduzierte sich die Anzahl auf $27 \%$ in den Jahren 1992/93. Produktbilanzen werden in anderen Bereichen angewendet: Insbesondere der Bereich der Untersuchung von Kunststoff(granulaten) als Halbfertigprodukte hat zugenommen (Studien von Boustead u. a. ). Weitere Bereiche, die leicht an Bedeutung zugenommen haben, sind elektrotechnische Produkte, Energie, Geschirr sowie Verkehr.

Daran ist abzulesen, daß Produktbilanz-Studien in neue Bereiche vorgedrungen sind, also eine

\begin{tabular}{|c|c|c|}
\hline \multicolumn{3}{|c|}{$\begin{array}{l}\text { Untersuchungsobjekte von } \\
\text { Produktbilanz-Studien 1974-1993 }\end{array}$} \\
\hline $\begin{array}{l}\text { Untersuchungs- } \\
\text { objekte }\end{array}$ & $\begin{array}{l}\text { Absolute } \\
\text { Anzahl }\end{array}$ & $\begin{array}{l}\text { Relative } \\
\text { Anzahl } \\
\end{array}$ \\
\hline $\begin{array}{l}\text { Abfall } \\
\text { Baumaterialien } \\
\text { Elektrotechnische } \\
\text { Produkte } \\
\text { Energie } \\
\text { Geschirr } \\
\text { Hygiene/Reinigung } \\
\text { Kunststoffe } \\
\text { Landwirtschaft } \\
\text { Papier- und Druck- } \\
\text { erzeugnisse } \\
\text { Verkehr } \\
\text { Verpackungen } \\
\text { Sonstiges }\end{array}$ & $\begin{array}{r}7 \\
11 \\
7 \\
21 \\
20 \\
7\end{array}$ & $\begin{array}{r}4,0 \% \\
9,0 \% \\
3,5 \% \\
5,5 \% \\
3,5 \% \\
10,5 \% \\
10,0 \% \\
3,5 \% \\
2,5 \% \\
4,0 \% \\
39,0 \% \\
5,0 \% \\
\end{array}$ \\
\hline SUMME & 200 & $100,0 \%$ \\
\hline
\end{tabular}


Diffusion stattgefunden hat. Der Rückgang des Verpackungsthemas hat mehrere Ursachen:

- Es wurden in den letzten Jahren eine Reihe von bedeutsamen repräsentativen Studien für das deutsche Umweltbundesamt und das schweizerische Bundesamt für Umwelt, Wald und Landschaft (BUWAL) erstellt. Dies hat zu einem gewissen Abwarteverhalten bei der deutschen Verpackungswirtschaft und den Anwendern geführt, die weniger eigene Studien in Auftrag gegeben haben und auf die Arbeiten des UBAs warten.

- Die Verpackungsverordnung hat "zudem einen gesetzlichen Rahmen geschaffen, der von den einzelnen Unternehmen auszufüllen war und weniger ein ökobilanziellen Absicherung bedurfte.

- Daneben dürfte auch ein gewisser »Sättigungsgrad « bei diesem Thema erreicht sein. Sofern man sich der These anschließt, daß die meisten Verpackungsstudien auch an den Endverbraucher gerichtet sind, so dominiert bisher der Bereich der Endprodukte und hier wiederum der Konsumgüterbereich. Demgegenüber wurden speziell Investitionsgüter kaum untersucht - Halbfertigprodukte häufiger, wobei jedoch der Bereich der Kunststoff(granulate) dominiert. Produktbilanzen sind aus dieser Sicht eher im Spannungsfeld zwischen Konsumgüterindustrie und Verbraucher angesiedelt als im Feld verschiedener Industriebranchen. Insofern gibt es noch ein großes Anwendungsfeld von Produktbilanzen in bisher »unerschlossenen« Bereichen. Gerade diese Bereiche dürften die »Normalität« industriell-stofflicher Beziehungen charakterisieren. Erst die Anwendung von Produktbilanzen in diesen Bereichen kann ihren eigentlichen Durchbruch bringen, denn sie dürften dann wesentlich mehr zur Optimierung der Stoff- und Energieflüsse beitragen als ihre Anwendung in Bereichen, die dem Marketinginteresse nahestehen.

Abbildung 1: Auftraggeber von Produkt-Bilanz-Studien

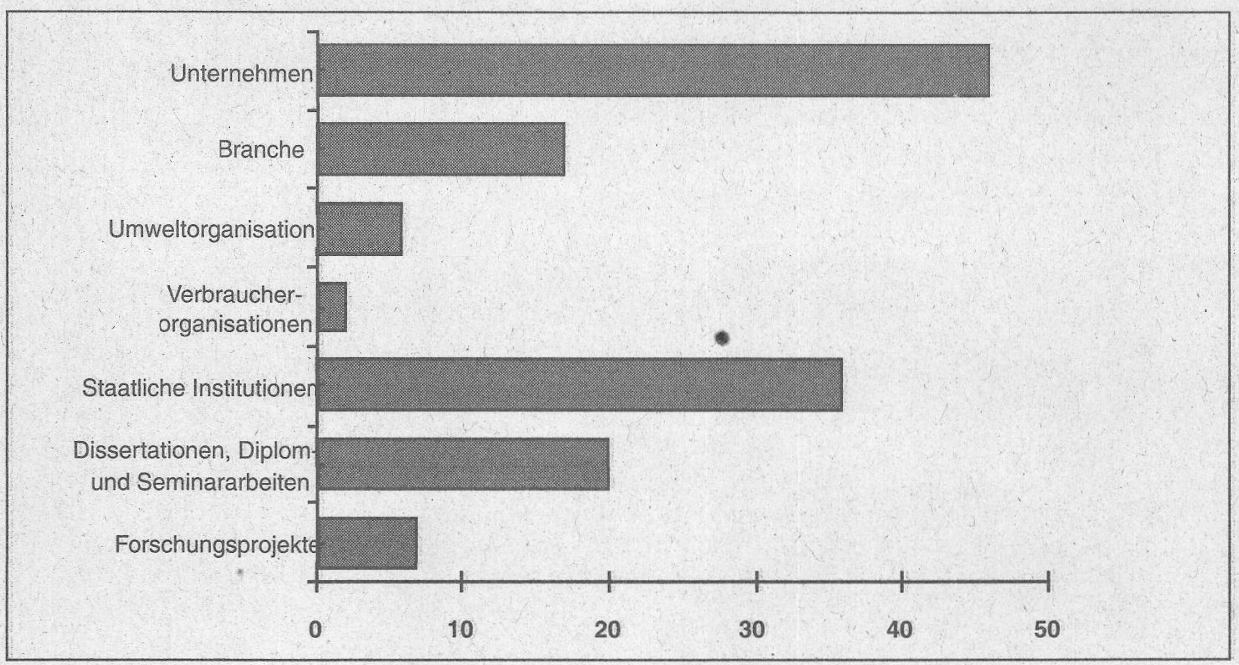

(Basis: $n=135$, da bei einer größeren Anzahl von Studien der Auftraggeber nicht identifizierbar ist)
Eine weitere Analyse hat auch die Auftraggeber von Produktbilanz-Studien zu erfassen. Auftraggeber können einzelne Unternehmen, ein Zusammenschluß mehrerer Unternehmen bis hin zur gesamten Branche, staatliche Institutionen, Umwelt- und Verbraucherorganisationen und Gewerkschaften sein. Daneben werden Produktbilanzen auch von Forschungseinrichtungen ohne speziellen Auftraggeber und im Rahmen von Seminar- und Diplomarbeiten sowie Dissertationen erstellt. Abbildung 1 zeigt, daß eindeutig Untersuchungen im Auftrage eines einzelnen Unternehmens oder mehrerer Unternehmen bzw. der gesamten Branche dominieren. Aber auch staatliche Institutionen haben eine ganze Reihe von Untersuchungen durchführen lassen. Umwelt- und Verbraucherorganisationen spielen bisher als Auftraggeber nur ein sehr untergeordnete Rolle.

Bisher ist es nicht bekannt geworden, daß Gewerkschaften Auftraggeber von Produkt-Ökobilanz-Studien gewesen wären. Dies ist wohl auch das Resultat einer gewissen Zurüickhaltung in den Gewerkschaften gegenüber diesem Instrument - zumindest was die Vergangenheit anbelangt.

Eine Unterscheidung kann auch nach der Repräsentativität bzw. Singularität einer Untersuchung erfolgen. Repräsentative Studien, die häufig auch als Parameterstudien bezeichnet werden, beziehen sich auf unternehmensund produktübergreifende Daten und können eine größere Repräsentativität in ihrer Aussagekraft beanspruchen. Fallstudien hingegen ermitteln spezifische Daten für den Lebenszyklus eines einzelnen Produktes auf der Basis unternehmens- und produktspezifischer Daten, sie beziehen sich also auf ein konkretes Beispiel eines Produktes eines Unternehmens. Fallstudien können deswegen nur Einzelfälle beschreiben und sind keineswegs repräsentativ. Bei der Analyse der uns verfügbaren Arbeiten zeigte es sich, daß nur eine beschränkte Anzahl

\section{da}

- neue Anwendungsbereiche zu erschließen (Investitionsgüterindustrie, Halbfertigprodukte),

- verstärkt repräsentative Daten für einzelne Bereiche einer Produktbilanz (u. a. Energie, Grundmaterialien, Abfall, Verkehr) zur Verfïgung zu stellen,

- methodische Unsicherheiten weiter abzubauen sowie

- den partizipativen Aspekt der Erstellung, Auswertung und Einbettung in Entscheidungsprozesse herauszuarbeiten und zu verfolgen (vgl. hierzu auch meine Thesen »Produktbilanzen - State of the art« - auf S. 14).

\section{Frieder Rubik, Heidelberg}

Dieser Beitrag wird in einer ausführlicheren und aktualisierten Version in dem Buch »Ökologische Produktpolitik « von Frieder Rubik und Volker Teichert erscheinen, das im Herbst 1994 veröffentlicht wird. 
(c) 20I0 Authors; licensee IÖW and oekom verlag. This is an article distributed under the terms of the Creative Commons Attribution Non-Commercial No Derivates License (http://creativecommons.org/licenses/by-nc-nd/3.o/), which permits unrestricted use, distribution, and reproduction in any medium, provided the original work is properly cited. 